\title{
Template Matching to Enhance HUD Colour Images
}

\author{
Shashi Poddar \\ CSIR-CSIO, Chandigarh, India- \\ 160030
}

\author{
Amitava Das \\ CSIR-CSIO, Chandigarh, India- \\ 160030
}

\author{
Vinod Karar \\ CSIR-CSIO, Chandigarh, India- \\ 160030
}

\begin{abstract}
Head up Display (HUD) is a means of presenting information to the pilot key flight instrument data onto a set of glasses regarded as 'beam combiner' positioned just in front of the pilot's line of sight looking ahead out of the aircraft helping him in key tasks like take off, navigation and landing. It also improves the situational awareness greatly by displaying flight, sensor and instrument data in the pilot's forward field of view. HUD employs a CCD camera mounted right in front of the pilot, that is, between the beam combiner and the pilot for recording the view comprising of the outside world and the HUD symbology as seen by the pilot. This recorded video is sent directly through telemetry to the flight analysis ground station for real time flight data analysis as seen by the pilot as well as to guide the pilot. The recorded video is also used for post flight analysis as well as video source for HUD repeater display used by the pilot occupying rear cockpit seat in case of trainer aircrafts. The inconsistent and improper lighting conditions prevailing during weather conditions such as rain, snow, fog, bright sunny daylight and against the white clouds causes either insufficient or excessive light intensity at different spatial locations falling on the CCD camera sensor head resulting in degraded image quality with very low contrast. The work presented in this paper attempts to tackle this problem through theoretical study and experimental analysis. A customized template matching technique is also presented here for enhancement of the video captured by HUD camera. Added to this, hardware based methods are also suggested which can be employed for real time video enhancement.
\end{abstract}

\section{General Terms}

Pattern recognition, Avionics display system

\section{Keywords}

Head Up Display, CCD imaging, Simultaneous contrast, Image segmentation, Template matching

\section{INTRODUCTION}

Head up display is an intrinsic part of modern aircraft cockpit assisting and augmenting the decision making capability of the pilot by displaying significant information on HUD. This helps in making more time available in looking through to the route ahead and helping in reducing the missing of targets. Thus it helps in a better elucidation of information with pilot's head always "up" and looking ahead instead of looking at instrument panels. The symbols are placed such that the pilot's view of external environment is minimally hindered and assistance in aircraft handling operations like, landing, etc. is enhanced. The data is gathered from various instruments in the cockpit and presented to the pilot in an easily perceivable form. The graphic display of all the meter readings is superimposed on the outside world on the HUD beam combiner glasses [1]. The Head-Up Display optically projects the image of the flight information at infinity so that the pilot can see all flight parametric information superimposed on the image of the real world. The information is actually displayed on a very high intensity display device and the symbology developed on the face of display device is collimated using complex set of optical glasses and mirrors and then projected using beam combiners. The beam combiner is made highly frequency selective to reflect only the display wavelength and to transmit all other visible wavelengths [2]. The display source may be a high brightness short length CRT, DLE or LCD to form the image. The HUD electronics provides interface with aircraft sensors and other systems and generate the symbology called as HUD symbology based on computations over the data received from various aircraft sensors and systems. The CCD Cameras used in HUD are traditionally placed at a location, which happens to be between beam combiners and the pilot, from where it can capture the same composite view as viewed by the pilot. The captured video can be used in rear cockpit display, the Ground Station for analysis as well as interpretation and also for post flight examination. The composite image obtained by the HUD camera forms the main source of information for pilots sitting in the rear cockpit. Hence, it is essential to provide a high quality image of what the flying pilot actually visualizes. Certain hardware limitations of the camera, however, results in a lower quality image. This is further compounded by the effect of background on the colour of foreground symbology leading to an added distortion in the captured image.

In section 2, the first part discourses the complexities and problems associated with CCD cameras. In section 3 few approaches have been suggested to overcome the problem and provide results and discussions for the proposed solution. Section 5 concludes the paper.

\section{PROBLEM DISCUSSION}

\subsection{CCD color camera related issues}

HUD CCD cameras have an auto-iris mechanism to adjust the aperture of the camera automatically, to control the amount of light entering the lens. The aperture opening is indirectly proportional to average intensity of light falling on the CCD pixels. However, a real world scene is very complex and the brightness values of the sensed area vary spatially. As a result, the aperture of the camera (which depends on the average brightness of the complete sensed area), may not provide proper integration time for very bright and dark area simultaneously. And this eventually leads to a low quality image and unnatural artifacts in the acquired image.

In order to avoid this problem, several researchers have made attempts to solve the problem in various ways. Few of them have tried to capture several images of the same scene using different exposures, to yield a high dynamic range image. Low exposure images provide enhanced view of the bright regions whereas high exposure images provide detailed view of the dark region. Use of beam splitters to generate multiple copies of the image, use of multiple potential well of different sizes and use of spatially varying pixel exposures of neighbouring pixels, are few of the other techniques used to implement high dynamic range imaging [3]. Recently, Nayar et al. suggested adaptation of the pixel exposure based on 
scene radiance by using an optical attenuator [3]. Furthermore, a patent has been filed by Nayar et al. which uses digital light processing for adaptive imaging of a scene [4]. However, these devices are still under development and needs further technology up-gradation to be employed for real world situation.

\subsection{Effect of background on foreground symbology}

Simultaneous contrast or induction is the influence on the perception of an object when visualized in the surrounding of other object [5]. For example, when a white disk is viewed on a red background, the disk appears to be tinged with the complement of red, that is, green [6]. This phenomenon is specifically termed as 'opponent chromatic induction'

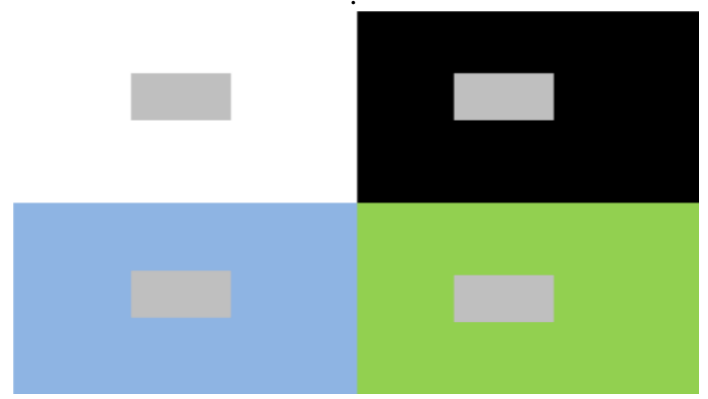

Fig 1: The simultaneous color contrast effect

Fig.1 shows four gray boxes of same color in the centre of each quadrant, but they appear to be of quite different color, because of the surrounding color. The brightness simultaneous contrast and chromatic simultaneous contrast has been studied in detail by many researchers and has been explained on the basis of optical processing done by the brain [7].

As seen in Fig. 2, it can be seen that the HUD symbology changes it color spatially depending on the color of the background. This image has been captured by using a background with sharp edges to emphasize the problem in a vibrant manner.

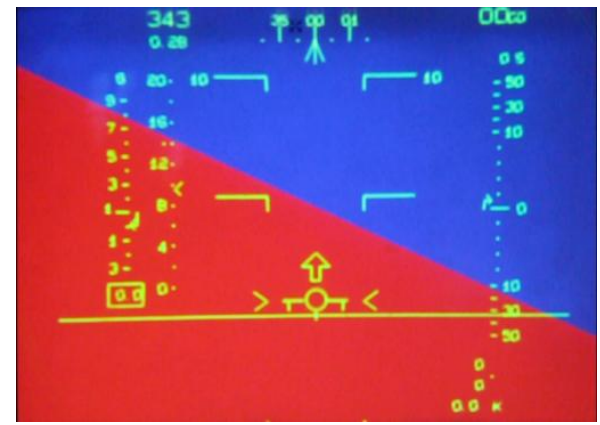

Fig 2: Showing change in symbology color with background

Fig. 2 is synthetically simulated in the lab. As observed, there are also certain extreme conditions, such as white background or bright sunlight, in which few symbols merge with the background. In order to overcome theselimitations, an algorithm has been presented in section 4, which reemphasizes the HUD symbology on the image captured by HUD camera.

\section{LITERATURE SURVEY}

There are several advancements, which are being looked upon in the field of better imaging systems, but still these improvements are limited by the pace of other technological improvements.

Optical attenuator based high dynamic range camera have increased size and has limitations in respect to scenes with very rapid motions [8]. In order to combat this problem, a technique of multiple iris based CCD system is being looked upon, which can alter the amount of light falling on the pixels depending upon the light intensity. A further advancement was sought after by changing the gain factor for each pixel depending on the rate of charge build-up within a particular pixel. However, this particular solution would not limit the saturation effect and may not result in highly improved quality of the image. Another solution to the problem of spatially varying light intensity imaging is to have a varying integration/exposure of pixels depending on the light intensity falling on them. Although, this seems to be a feasible solution, it is limited by the computational overhead required in real time video capture. It requires several comparisons and closed loop feedback control mechanism to be implemented within few nanoseconds which is not really achievable with present electronics system.

Owing to the reasons discussed in section 2, the quality of the image produced by the HUD cameras is poor and the information displayed, are not distinct from the background image. Hua bao et al. [9] proposed a technique of contrast enhancement followed by adaptive thresholding for recognizing the characters embedded in the image. This work employs the adaptive thresholding technique, proposed originally by Huang et al. [10]. This has certain problems with its implementation for character recognition of HUD images as explained below.

Adaptive thresholding [10] divides the image into several subimages wherein the size of the window varies on the basis of Lorentz information measure. These sub-images are then segmented separately using Otsu's thresholding algorithm, to furnish an image with high contrast between the background and the object. The implementation of this technique on the HUD images yields an image with spurious results. As seen in Fig. 3, the clouds in these images are treated as foreground object (shown under red circle) rather than background, and results in the ambiguous outcome of the processed image.

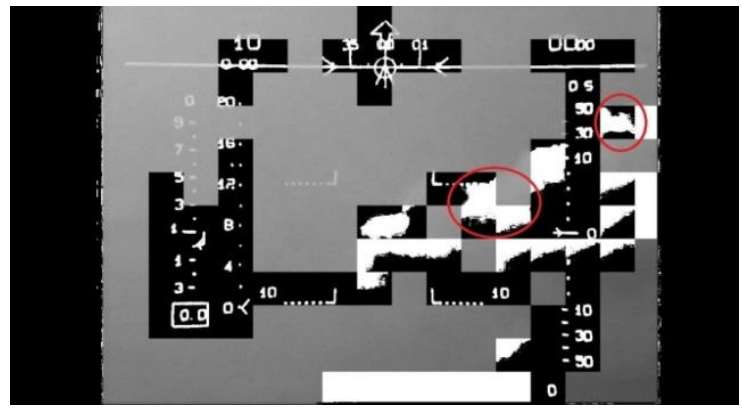

Fig 3: Showing results of adaptive thresholding algorithm implemented in [10]

Image Segmentation with windows of fixed size resulted in similar problems as shown in the figure below. 


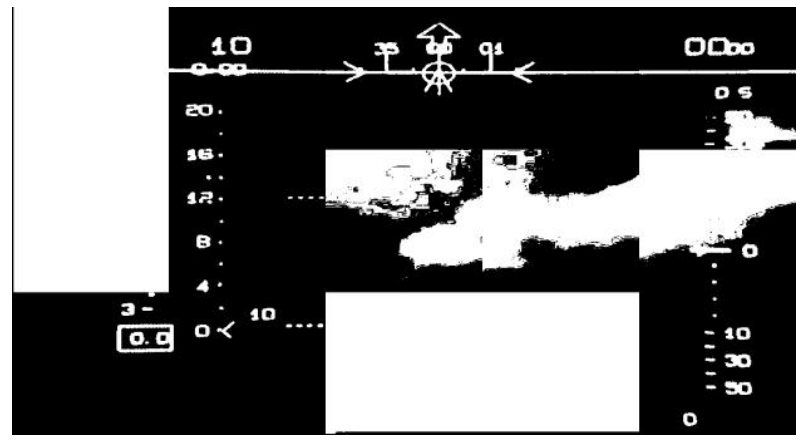

Fig 4: Showing the Thresholding result with fixed window size

With these un-satisfactory results, a template matching algorithm was employed to recognize the characters present in the image [11].

\section{ALGORITHM}

The algorithm developed here is implemented for each frame in the video sequence. A set of synthetic template was initially extracted from the standard image sets, which were also corrected for any distortions manually. Fig. 5 shows the set of static template used for matching in the image and Fig. 6 shows the dynamic templates which change their position value with time. The algorithm for the proposed methodology is presented below as:

1. Let us consider a color image $\{I=I(i, j, 1: 3) \mid 1<$ $i<M, 1<j<N\}$ of size $M \times N \times 3$.

2. A set of all possible symbols is synthetically developed with sharp edges, with ' 0 ' representing the background and ' 255 ' representing the foreground object.

3. These templates are divided into two categories i.e. 1) Spatially fixed symbols 2) spatially varying symbols.

4. The color image is then converted to gray scale image $\left(\mathrm{I}_{\mathrm{g}}\right)$ by averaging all three $\mathrm{R}, \mathrm{G}$, and $\mathrm{B}$ scales.

5. For templates that do-not change their position, but change their numeric value alone, is matched from 0-9 for specific positions and frames. Although the search area is specific, a small tolerance of 2 pixels is kept on all sides for any small change.

6. The normalized cross-correlation is calculated for each incremented position of template mask (T) and a sub-image extracted from the original gray scale image $\left(\mathrm{I}_{\mathrm{g}}\right)$.

7. The value corresponding to the maximum correlation and a value greater than 0.8 is considered to be the true match between the template and sub-image.

8. The position of match is then saved in another variable ' $M$ ' such that $\mathrm{T}(\mathrm{i}, \mathrm{j})=255$.

9. With these saved locations in $M$, the change is transferred to all the three R, G and B scales such that $I(i, j, 1)=150 ; I(i, j, 2)=250 ; I(i, j, 3)=150$ where $(i, j) \in M$.

10. For templates, that are static and even do not change in numerals are matched directly and steps 6-9 is repeated for them directly.

11. For symbols that vary with position, are needed to be searched in the complete image. Hence, the initial gray scale image is resized to a smaller size and a template matching is applied with the template resized with the same scale. (Scale chosen in the algorithm developed is $1 / 8$ ).

12. After a match is found by applying steps $6-8$, the location is scaled for the original gray scale image, and a local matching is done for precise location of template in the image by the same method.

13. Step 9 then follows this step for transformation in all the three scales.

14. Templates that rotate dynamically, is matched for different angles in steps of 3 degrees, and is also done in reduced scale image for faster operation. Steps 6-9 follow the sequence similarly.

15. Angular value of the matched template is stored in another variable 'angle' for its use in sequent frames to estimate the orientation of template in the forthcoming frames.

16. Steps $1-15$ are then followed for each frame in the video sequence.
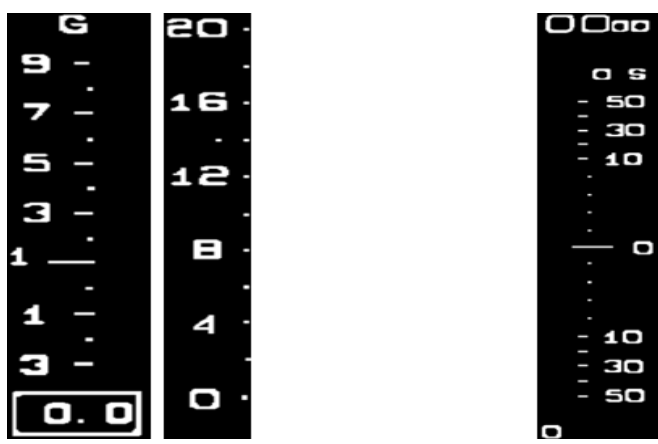

Fig. 5 Synthetic static templates
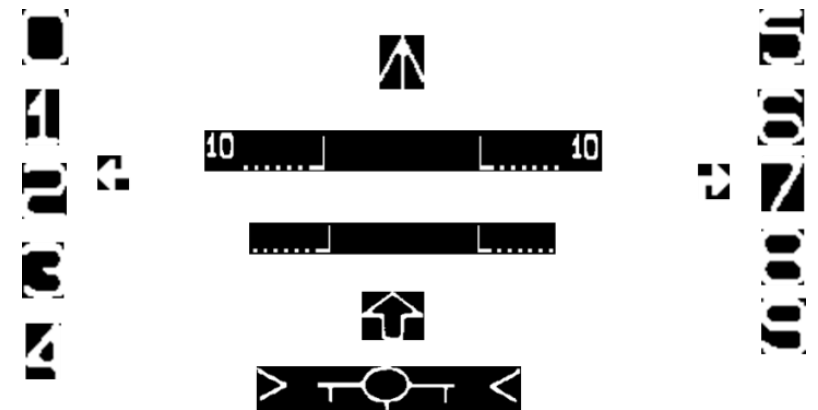

Fig. 6 Synthetic dynamic templates

\section{RESULTS AND DISCUSSION}

The result obtained after applying the algorithm on an image discussed in section 4 is presented in Fig. 5. The algorithm developed in section 4 were also employed for a small video sequence and showed promising result in the enhancement of the quality of displayed symbology.

Though a modification has been done only in the foreground symbology, it also leads to an overall increase in the contrast of the image. The symbols which seemed to be embedded in the background are sharp and have better appearance as compared to the original image. A further improvement, in the algorithm developed in section 4 was done by including a sense of knowledge to predict the symbol in sequent frames.

The position and the angle of a symbol, which change dynamically with time, are stored in a local variable. This 
orientation value is then used to predict the position of the corresponding symbol in the next frame. This reduces the search time for the best match and fastens the algorithm.

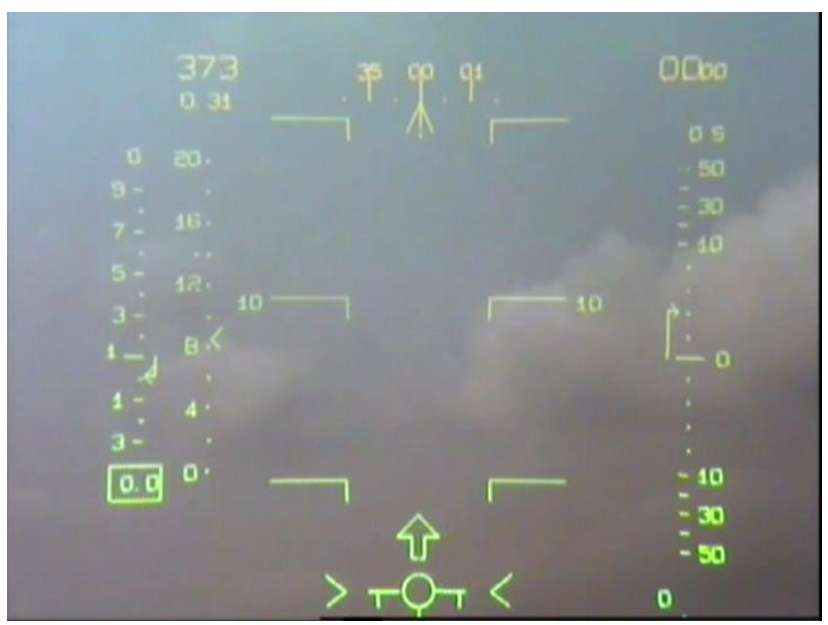

(a)

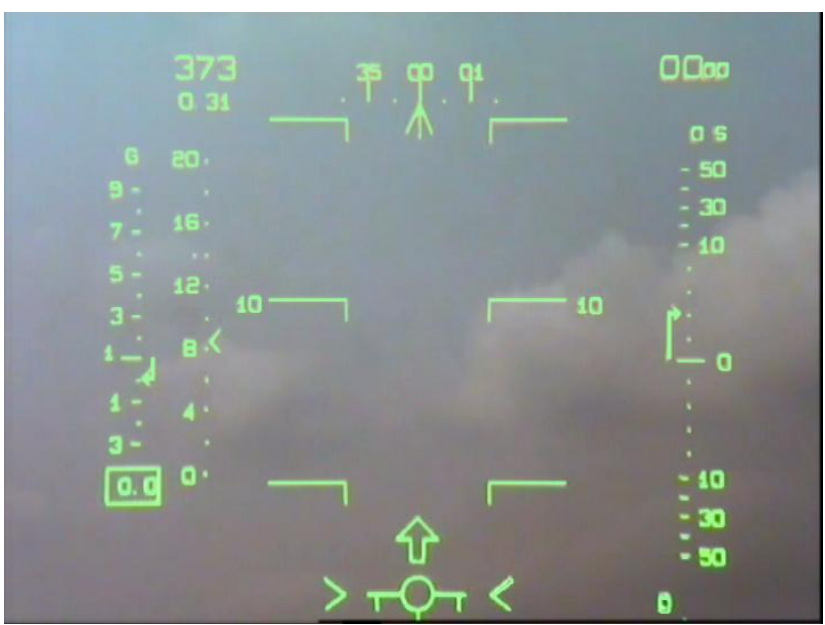

(b)

Fig7: (a) Original image captured by HUD camera (b) Modified image by applying the proposed algorithm

Added to it there are certain cases when a particular symbol is hidden in the background, but has a fixed relationship with another template which is easily decipherable in the image. In these cases, the fixed relationship is used to predict the location of the template and a superimposition of the template is done over the image.

\section{CONCLUSION \& FUTURE WORK}

In this work, an algorithm using template matching was developed to automatically detect and make transformation in the original color image for improving the contrast of the low quality image captured by HUD camera. The results shown above clearly indicate the improvement in the image quality captured by camera. For video sequence, a sense of knowledge is included in the algorithm to predict the symbol and its position based on previous sequences. The prediction algorithm needs to be strengthened further to improve its efficiency and time usage. Presently this algorithm is applied only for the offline video enhancement, but improvements are still being looked after to implement it for real time online image enhancement. Added to this, hardware based methods are also suggested which can be employed for real time video enhancement.

\section{ACKNOWLEDGEMENT}

The authors would like to thank CSIR for giving an opportunity and the support for carrying out the research work. The author wishes to acknowledge Dr. Pawan Kapur, Director (CSIO) for motivation and providing a conducive environment for research. Authors would also like to acknowledge Prof. Sankar K. Pal and Prof. Ashish Ghosh at ISI Kolkata for their support and guidance.

\section{REFERENCES}

[1] Available [Online] http://en.wikipedia.org/wiki/Headup_display

[2] Marconi, U.S. Patent 4,261,647 (1981), Head Up Displays

[3] S. K. Nayar and V. Branzoi, "Adaptive dynamic range imaging: optical control of pixel exposures over space and time". In Proc . ICCV, volume 2, p ages 11681175, Oct 2003.

[4] Nayar et. Al, "Adaptive imaging using digital light processing”, US Patent, 0211077, Sep. 2011.

[5] Michael A. Webster, J.D. Mollon, The influence of contrast adaptation on color appearance, Vision Research, Volume 34, Issue 15, August 1994, Pages 1993-2020, ISSN 0042-6989, 10.1016/00426989(94)90028-0.

[6] Hsien-Che-Lee, "Introduction to color imaging science", pg. 354-pg357, Cambridge University Press, Cambridge (2005).

[7] Purves, D. \& Lotto, R. B. (2003) Why We See What We Do: An Empirical Theory of Vision (Sinauer, Sunderland, MA).

[8] Mannami, H.; Sagawa, R.; Mukaigawa, Y.; Echigo, T.; Yagi, Y.; , "High Dynamic Range Camera using Reflective Liquid Crystal," Computer Vision, 2007. ICCV 2007. IEEE 11th International Conference on , vol., no., pp.1-8, 14-21 Oct. 2007

[9] H. Bao, G. Wang, N. Li, "Character Recognition of Instrument Board in Sequent Color Image", IS\&T/SPIE's 15th Annual Symposium on Electronic Imaging, Santa Clara, California, U.S.A, Jan. 2003

[10] Qingming Huang, Wen Gao, WenjianCai, Thresholding technique with adaptive window selection for uneven lighting image, Pattern Recognition Letters, Volume 26, Issue 6, 1 May 2005, Pages 801-808.

[11] R.C.Gonzalez and R.E.Woods, "Digital Image Processing', 3rd edition, Prentice Hall, 2009 